\title{
Creation of chiral adsorbed structures using external inputs: results from lattice Monte Carlo simulations
}

\author{
Aleksandra Woszczyk ${ }^{1} \cdot$ Paweł Szabelski $^{1}$
}

Received: 12 October 2015/Revised: 8 November 2015/Accepted: 21 November 2015/Published online: 28 November 2015

(C) The Author(s) 2015. This article is published with open access at Springerlink.com

\begin{abstract}
Patterning of solid surfaces with organic molecules has been recognized as a promising method to create functional 2D matrices with tunable structure and properties. In this work we use the lattice Monte Carlo simulations to study chiral pattern formation in adsorbed systems comprising simple molecular building blocks differing in shape. To that end we consider five-membered rigid isomers whose composite segments can occupy vertices of a triangular lattice and interact with short-range (nearest neighbors) forces. Our main focus is on those molecules which are prochiral, that is they can adopt mirror-image planar configurations when adsorbed. Moreover, the effect of orientational in-plane confinement of the molecules, which reflects their coupling with an external directional field, on the structure formation and chiral resolution in 2D is explored. The obtained results demonstrate that the confinement imposed on the surface enantiomers can induce their resolution and formation of extended homochiral domains. However, it is also shown that for certain molecular shapes the confinement can produce mixed racemic crystals instead of the homochiral assemblies. The insights from our simulation studies can be helpful in preliminary screening of molecular libraries to select optimal building blocks able to self-assembly into chiral 2D patterns with predefined architecture.
\end{abstract}

Keywords Chiral resolution - Adsorbed overlayers . Monte Carlo simulations · Prochiral molecules

Paweł Szabelski

szabla@vega.umcs.lublin.pl

1 Department of Theoretical Chemistry, Maria-Curie Skłodowska University, Pl. M. C. Skłodowskiej 3, 20-031 Lublin, Poland

\section{Introduction}

Separation and purification of chiral substances has been of central interest in pharmaceutical, food and agricultural industries. The main reason for the fabrication of enantiopure compounds is that mirror-image molecular structures often used as drugs, food and cosmetics additives etc. can have dramatically different pharmacological activities, for example one enantiomer being therapeutic while the other being toxic. To eliminate potential contact with the harmful enantiomer it is necessary to produce enantiopure compounds or to effectively separate components of racemic mixtures. In the latter case, chiral environments, for example, chiral stationary phases are usually used, providing selective enantiomer recognition (Ahuja 2000). To separate enantiomers or to direct their stereoselective synthesis chiraly templated surfaces have been recently proposed. Those surfaces comprise a solid support on which chiral modifier molecules are preadsorbed, and due to the 2D self-assembly they form a homochiral matrix able to interact selectively with the complementary guest enantiomers (Zaera 2008; Rampulla and Gellman 2004). In fact, it has been demonstrated in numerous cases that to create homochiral adsorbed structures one does not to have to use chiral modifier-conversely, even achiral molecules have been found to be able to create chiral 2D assemblies (Kudernac et al. 2009; Miyake et al. 2008; Mu et al. 2008). The question how extended is the obtained adsorbed structure and what is its architecture depends strongly on the shape and chemistry of the building block. To date different molecular bricks have been used to create such homochiral surface-supported assemblies, including chiral and achiral star-shaped organic molecules (Kudernac et al. 2009; Miyake et al. 2008; Mu et al. 2008; Tahara et al. 2011; Xu et al. 2013). 
A special kind of molecular building blocks are the prochiral molecules which are not chiral in bulk phase but when adsorbed they can exist in one of two mirror-image configurations. Normally, when no chiral bias is applied, the surface enantiomers are equally populated and depending on molecular properties such as shape and functional groups the enantiomers can form mixed structures or they can split. In both cases the composition of the overlayer is racemic on average but the structure is completely different. The formation of homochiral structures in the assemblies comprising one component that is achiral in 3D is particularly attractive from the practical point of view. Namely, in this case no enantiopure substances are needed, whose production and purification is usually tedious. For that reason, identification of factors which enhance such 2D chiral resolutions has been of great importance for designing and optimization of new methods to produce homochiral environments. One of such factors has been the reduction of rotational freedom of adsorbed molecules, induced for example by external directional fields.

As it has been recently demonstrated both experimentally (Berg and Patrick 2005; Mougous et al. 2000) and theoretically (Szabelski and Woszczyk 2012; Woszczyk and Szabelski 2013,2015) the orientational confinement imposed on adsorbing molecules can have great impact on their organization in surface-supported self-assembled structures. For example, using the simplified MC model we showed for small prochiral molecules that their effective segregation can be achieved when the molecules are forced to adopt unique in-plane orientation. This effect was observed previously for the four- and five-membered building blocks adsorbed on a square lattice (Szabelski and Woszczyk 2012; Woszczyk and Szabelski 2013, 2015) and for the four-membered molecules adsorbed on a triangular lattice. In the last case our investigations were limited to three cases as only three four-membered chain conformations produce prochiral tectons. For that reason, herein we extend our studies and focus on bigger molecules for which the number of possible chain conformations is larger, allowing for better understanding of the relation molecule2D superstructure. Specifically, in this contribution we use the MC simulation method to explore the role of chain conformation/ramification of flat rigid prochiral molecules on their phase behaviour in racemic $2 \mathrm{D}$ assemblies under orientational confinement. To that end we consider a set of molecular structures which resemble fused polyaromatic hydrocarbons and which are planar polyhexes comprising five hexagons arranged with adjacent sides. The main objective of our study is to identify those molecular building blocks which segregate easily producing enantiopure domains and those for which mixed 2D crystals are formed.

\subsection{Simulations}

To explore chiral ordering in adsorbed phases different theoretical methods have been used including Monte Carlo (MC) (Popa and Paci 2013; Perusquía et al. 2005; Martínez-González et al. 2014; Paci et al. 2007) simulations and statistical-mechanical calculations (Medved' et al. 2009, 2010, 2015). In this work we rely on the first method and build a MC model of the surface-confined self-assembly of simple five-membered prochiral molecules. It is assumed that these molecules are flat rigid structures composed of identical segments arranged in different shapes which are non-superimposable with their mirror images. Each of the composite segments can occupy one site on a triangular lattice. There are 22 possible arrangements of the segments leading to structurally different molecules on a triangular lattice. However, only 11 out of them are prochiral. These structures are schematically shown in Fig. 1.

In the following we consider the molecules from Fig. 1 and assume than they can interact via a short range attractive segment-segment interaction potential limited to nearest neighbors on a triangular lattice. Specifically, we assume that the energy of interaction between adjacent segments of a pair of molecules is characterized by the parameter $\varepsilon$ expressed in $k T$ units. The simulations were performed with the help of the lattice gas MC method with Metropolis sampling (Romá et al. 2003; Ramirez-Pastor et al. 1995) which was used in our previous studies (Szabelski and Woszczyk 2012, Woszczyk and Szabelski 2013, 2015). A detailed description of this method and the simulation algorithm can be found therein. In the calculations we used a 100 by 100 rhombic fragment of a triangular lattice and imposed periodic boundary conditions in both planar

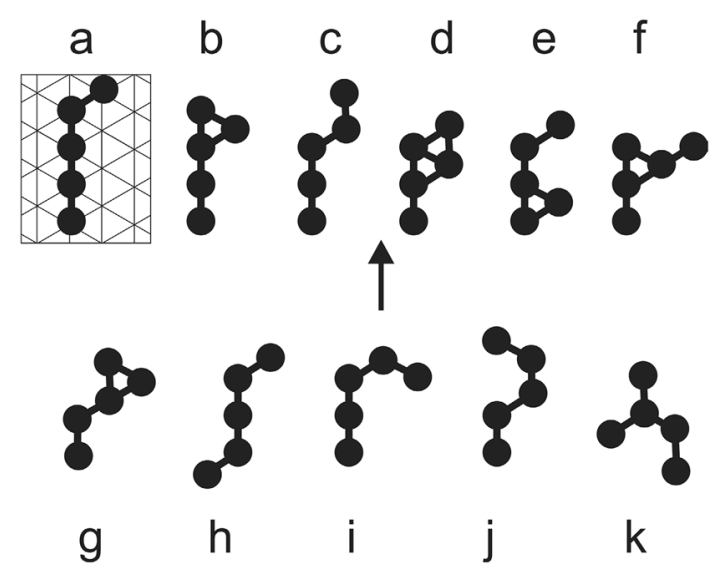

Fig. 1 Schematic structure of the model prochiral molecules used in the simulations. Only one surface enantiomer of each molecule, called arbitrarily $\mathrm{S}$ is shown. For molecule a mapping of the composite segments on a triangular lattice is additionally presented. The black arrow shows the assumed orientational confinement direction 
directions. To equilibrate the investigated systems we used typically $10^{9} \mathrm{MC}$ steps where one step is a single attempt to move each adsorbed molecule to a new position on the lattice. Additionally we applied the annealing procedure during which the adsorbed overlayer was cooled down in a linear fashion from $T=2$ to $T=0.2$ within $20 \mathrm{MC}$ steps intervals of equal length. All of the calculations were performed for 1300 molecules, for $\varepsilon=-1$. Two qualitatively different situations were considered in which (1) the molecules were allowed to take any of the six planar orientations imposed by the symmetry of the triangular lattice and (2) the molecules were confined, being able to adopt only one planar orientation marked by the black arrow in Fig. 1. These two cases are further called isotropic and unidirectional model, respectively.

\section{Results and discussion}

\subsection{Enantiopure assemblies}

To examine the effect of the oriantational confinement on the structure formation in our systems we first performed the simulations for the enantiopure overlayers comprising the 1300 molecules from Fig. 1. Figure 2 presents exemplary snapshots obtained for the tectons $\mathbf{a}, \mathbf{b}, \mathbf{j}$ and $\mathbf{k}$ when all of the in-plane molecular orientations were allowed (isotropic model). These molecules were selected because they differ significantly in shape, aspect ratio and ramification embracing a wide range of structural features.
As it can be seen in the figure, for any of these molecules we did not observe spontaneous ordering leading to extended fully periodic patterns. Instead, compact aggregates comprising locally ordered molecular clusters were only formed. This local ordering is particularly visible for the molecule a for which lamellar patterns can be easily identified. Moreover, for molecule $\mathbf{j}$ we were able to identify ordered domains with the complex molecular packing and parallelogram $(\sqrt{7} \times \sqrt{13})$ unit cell shown in the inset to the corresponding panel. For this molecule diversified structural motifs were found inside the entire assembly and interestingly no hole effects occurred therein. Formation of disordered structures similar to these shown in Fig. 2. was observed for all of the remaining molecules, regardless of their chain conformation (results not shown).

The above situation changes drastically when the orientational confinement is switched on. Figure 3 shows the snapshots obtained for the same set of molecules $(\mathbf{a}, \mathbf{b}, \mathbf{j}$ and $\mathbf{k}$ ) but for the unidirectional model. In this case we can observe emergence of ordered structures in which the molecules form periodic patterns aligned parallel to the guiding factor. These patterns are characterized by the following parallelogram unit cells which we marked in the figure, that is $\mathbf{a}(1 \times 5), \mathbf{b}(\sqrt{3} \times \sqrt{7}), \mathbf{j}(3 \times \sqrt{13})$ and $\mathbf{k}$ $(2 \times 3)$.

\subsection{Racemic assemblies}

To compare the results obtained for the enantiopure systems with their racemic counterparts we performed

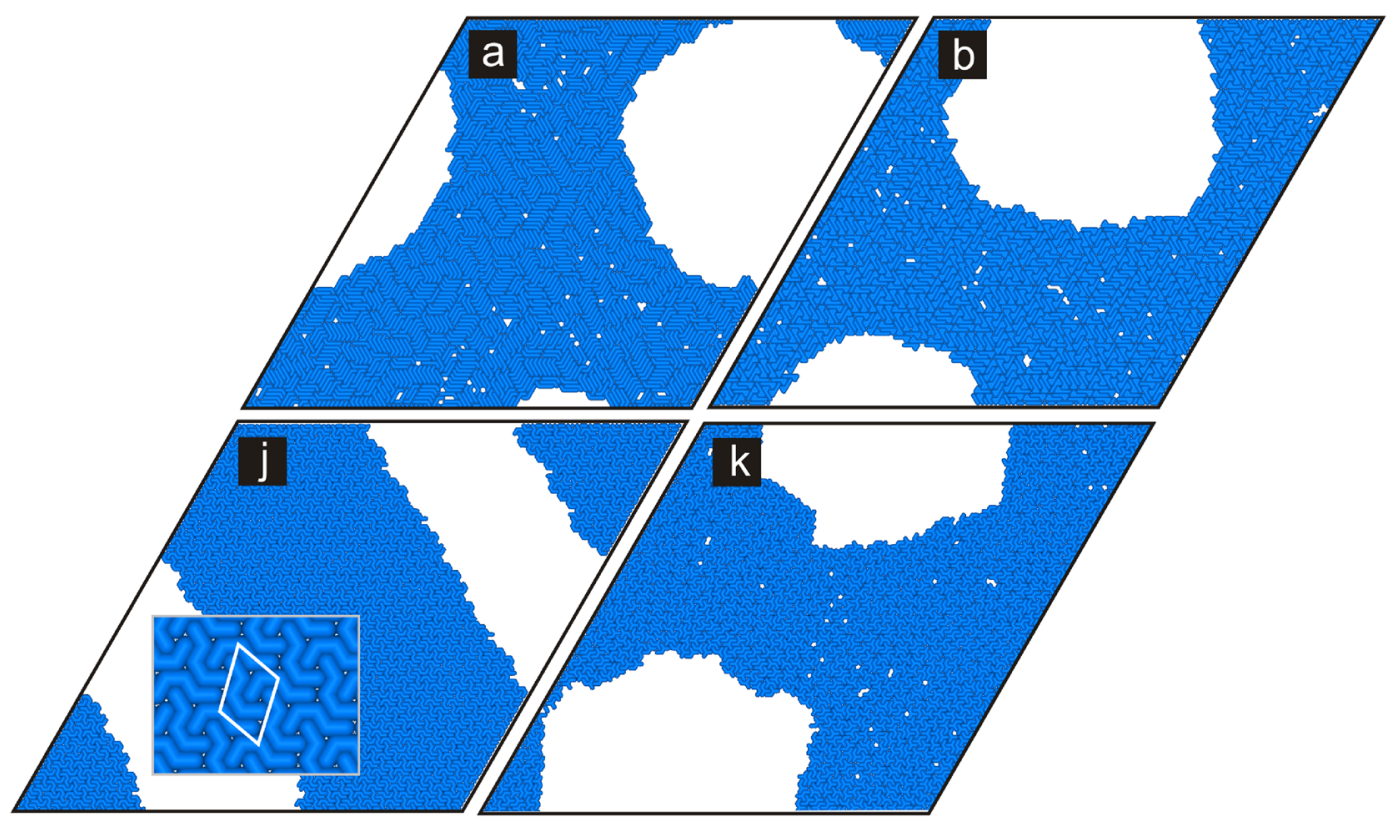

Fig. 2 Snapshots of the enantiopure overlayers (S) simulated for 1300 molecules of $\mathbf{a}, \mathbf{b}, \mathbf{j}$ and $\mathbf{k}$, for the isotropic model. The inset in panel $\mathbf{j}$ presents a fragment of the complex ordered domain characterized by the parallelogram unit cell shown in white 


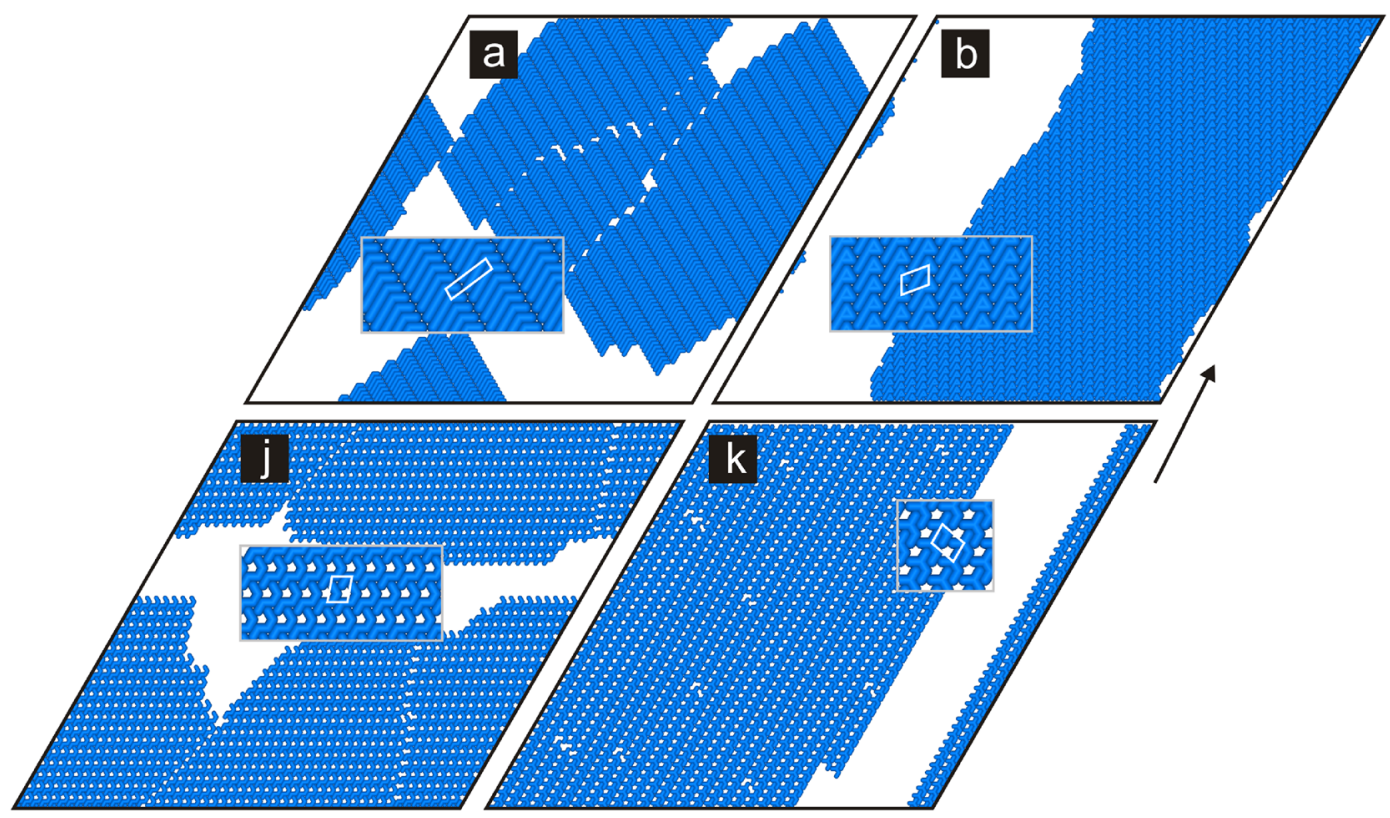

Fig. 3 Snapshots of the enantiopure (S) overlayers simulated for 1300 molecules of $\mathbf{a}, \mathbf{b}, \mathbf{j}$ and $\mathbf{k}$, for the unidirectional model. The insets show magnified fragments of the adsorbed superstructures whose unit cells were marked in white

additional calculations in which $650 \mathrm{~S}$ and $650 \mathrm{R}$ enantiomers of $\mathbf{a}, \mathbf{b}, \mathbf{j}$ and $\mathbf{k}$ were mixed. Figure 4 presents the simulated snapshots.

In the case of the racemates we can observe that the presence of the other enantiomer has a very little effect on the extent of ordering in the simulated systems when no bias is applied. Comparison of the obtained data with those from Fig. 2 proves that for both types of systems (enantiopure vs. racamic) the self-assembled structures are very similar. Specifically, in the racemic assemblies we can also identify small randomly oriented molecular clusters which are tightly packed to form a compact domain.

The most interesting findings were, however, obtained for the racemates whose components were orientationally confined. Figure 5 presents the snapshots simulated for the molecules $\mathbf{a}, \mathbf{b}, \mathbf{j}$ and $\mathbf{k}$, for the unidirectional model. As it

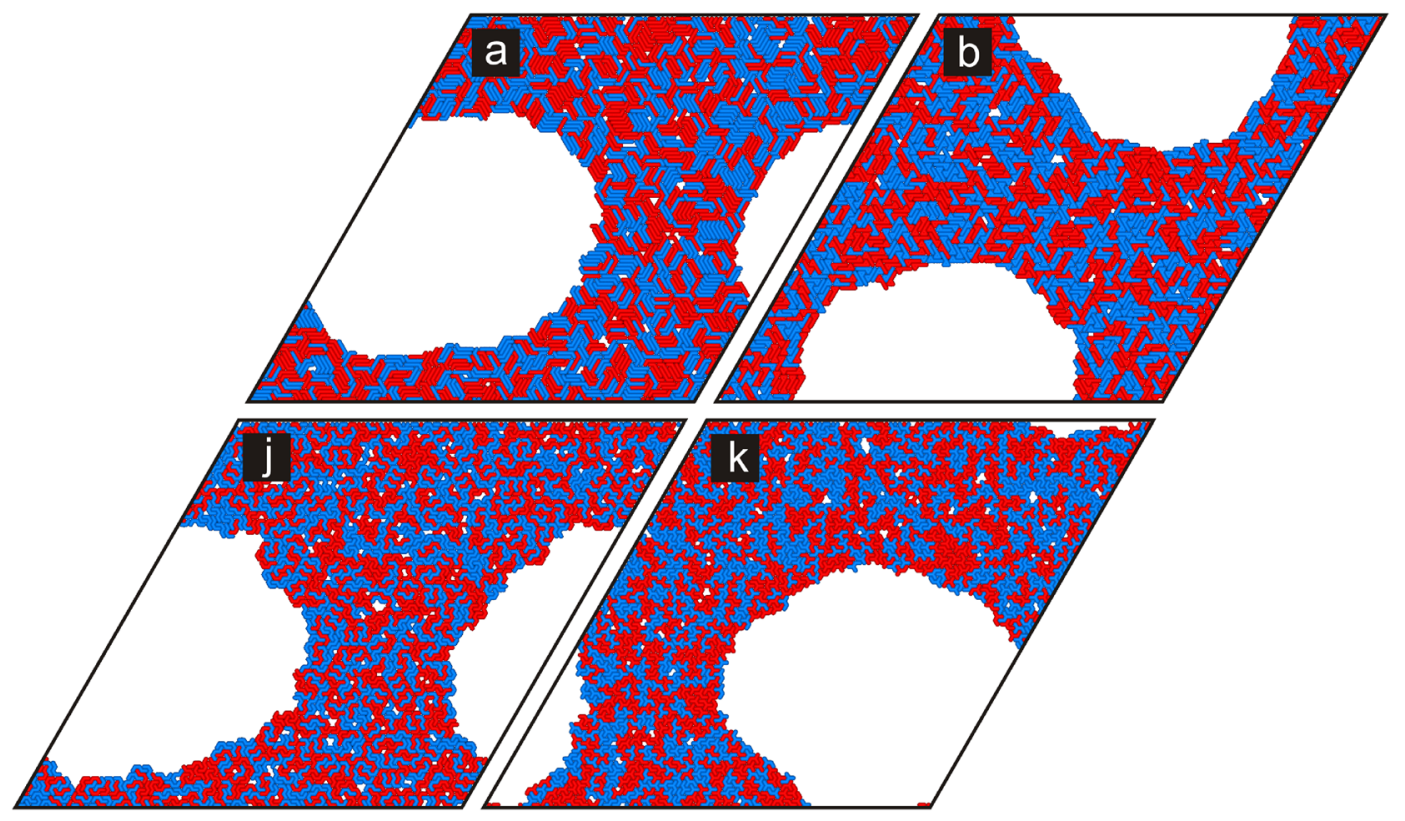

Fig. 4 Snapshots of the racemic overlayers simulated for 1300 molecules of $\mathbf{a}, \mathbf{b}, \mathbf{j}$ and $\mathbf{k}$, for the isotropic model 
is clearly seen in the figure, the two molecules a and b exhibit totally different phase behaviour than the remaining pair $(\mathbf{j}, \mathbf{k})$. In the first case we are dealing with complete enantiomer resolution resulting in extended homochiral domains while in the latter case ordered mixed 2D crystals are formed.

The magnified fragments of these mixed structures are shown in Fig. 6. The 2D mixed crystals of $\mathbf{j}$ and $\mathbf{k}$ have a racemic (1:1) composition being in agreement with the presumed stoichiometry of the mixture. Analogous, racemic crystals were obtained for molecules $\mathbf{e}, \mathbf{f}, \mathbf{g}$ and $\mathbf{i}$ and the corresponding magnified fragments of the $2 \mathrm{D}$ crystals are shown in Fig. 6. The obtained mixed phases are characterized by the following unit cells: e $(\sqrt{7} \times 7)$, f $(\sqrt{7} \times 4), \mathbf{g}(2 \times 5), \mathbf{i}(3 \times 2 \sqrt{3}), \mathbf{j}(3 \times 4)$ and $\mathbf{k}(2 \times 5)$. We note that the above unit cells correspond to the domains which were found to be most prevalent in the corresponding adsorbed phases. These domains, when in contact, produced local structures with different periodicities and for the molecule $\mathbf{i}$ partial demixing leading to the homochiral domains occurred. In the remaining cases, that is for molecules $\mathbf{c}, \mathbf{d}$ and $\mathbf{h}$ we observed the chiral segregation and the creation of extended enantiopure domains (results not shown)

To quantify the extent of segregation in the orientationally confined racemates we used the parameter $h$ which provides the average number of heterogeneous segmentsegment R-S bonds in the adsorbed phase. Precisely, $h$ is the number of bonds that can be drawn to connect each segment of a molecule, say $\mathrm{R}$, with neighboring segments belonging to molecule $\mathrm{S}$. In a mixture whose components segregate this parameter should be close to zero while for mixing it can take other, non-zero, values, depending on the actual composition/periodicity (or its lack) of the selfassembled structure. In Fig. 7 we plotted the parameter $h$ as a function of the MC steps for all of the molecular structures $\mathbf{a}-\mathbf{k}$ considered in this work.

As it can be seen in the left panel of the figure, for molecules $\mathbf{a}, \mathbf{b}, \mathbf{c}, \mathbf{d}$ and $\mathbf{h}$ the average number of heterogeneous interactions drops to about zero in the course of the simulation. This can be observed in the interval from 5 to $7 \times 10^{8} \mathrm{MC}$ steps which corresponds to temperature interval 2-2.8. The observed effect is a clear manifestation of the segregation occurring in the adsorbed phase-leading to the homochiral domains some of which $(\mathbf{a}, \mathbf{b})$ are shown in Fig. 5. For the remaining molecules, however, the obtained limiting values of $h$ are significantly larger than zero which means the formation of the mixed patterns from Fig. 6. To demonstrate this, let us consider the values of the parameter $h$ calculated for the ideal defect-free structures shown in this figure. These are equal to 2.8 (e), 2.2 $(\mathbf{f}), 2.0(\mathbf{g}), 2.2(\mathbf{i}), 3.0(\mathbf{j}), 2.0(\mathbf{k})$. As it can be seen in Fig. 7, at the end of the simulation run all of the curves are close to the corresponding limiting values listed above. For all of the molecules the simulated values are somewhat lower than the theoretically predicted ones and this results directly from the presence of domain boundaries at which the molecules are not fully coordinated. A noticeable exception is the molecule $\mathbf{i}$, for which the simulated value of $h$ (about 1.5) is considerably smaller than the predicted

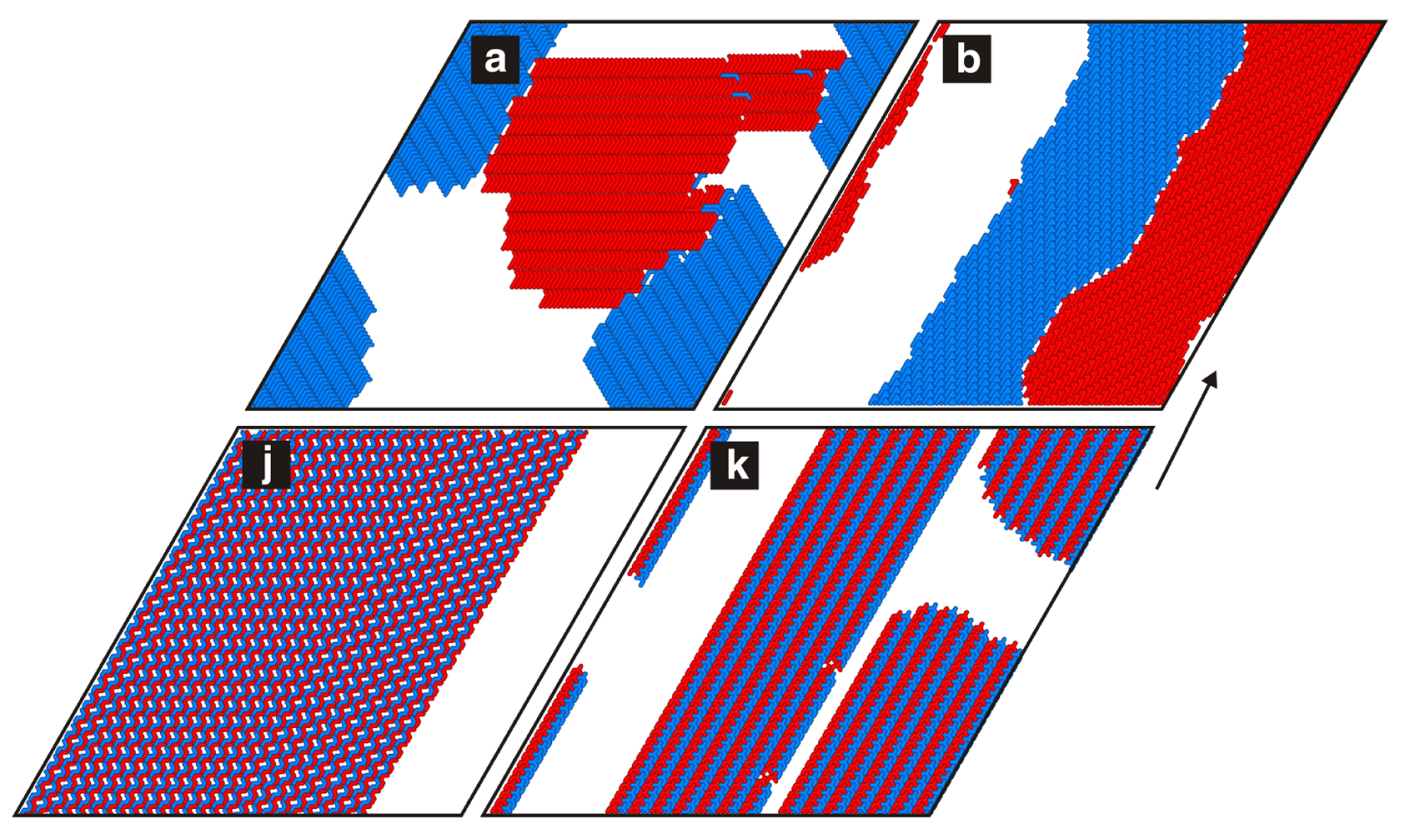

Fig. 5 Snapshots of the racemic overlayers simulated for 1300 molecules of $\mathbf{a}, \mathbf{b}, \mathbf{j}$ and $\mathbf{k}$, for the isotropic model. The black arrow indicates the orientational confinement 

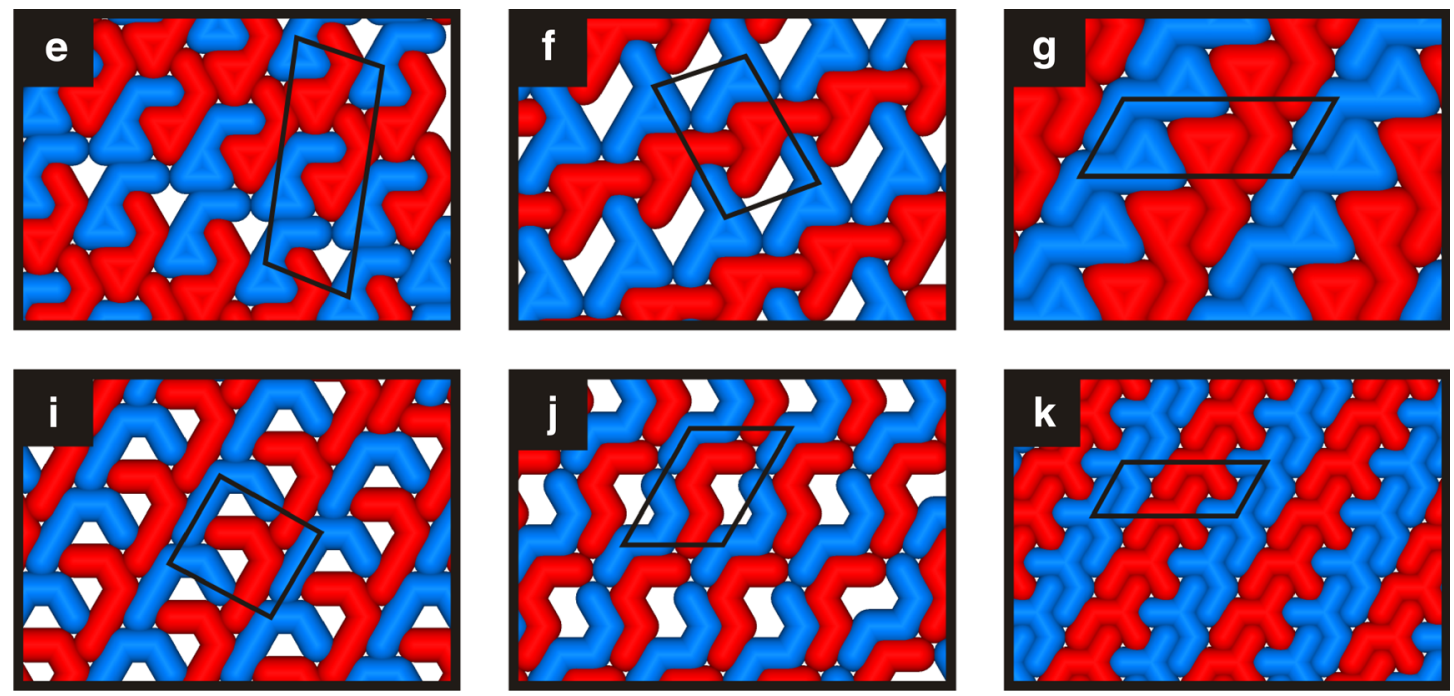

Fig. 6 Magnified fragments of the mixed structures formed by the molecules of $\mathbf{e}, \mathbf{f}, \mathbf{g}, \mathbf{i}, \mathbf{j}$ and $\mathbf{k}$. For each structure the corresponding unit cell is marked in black

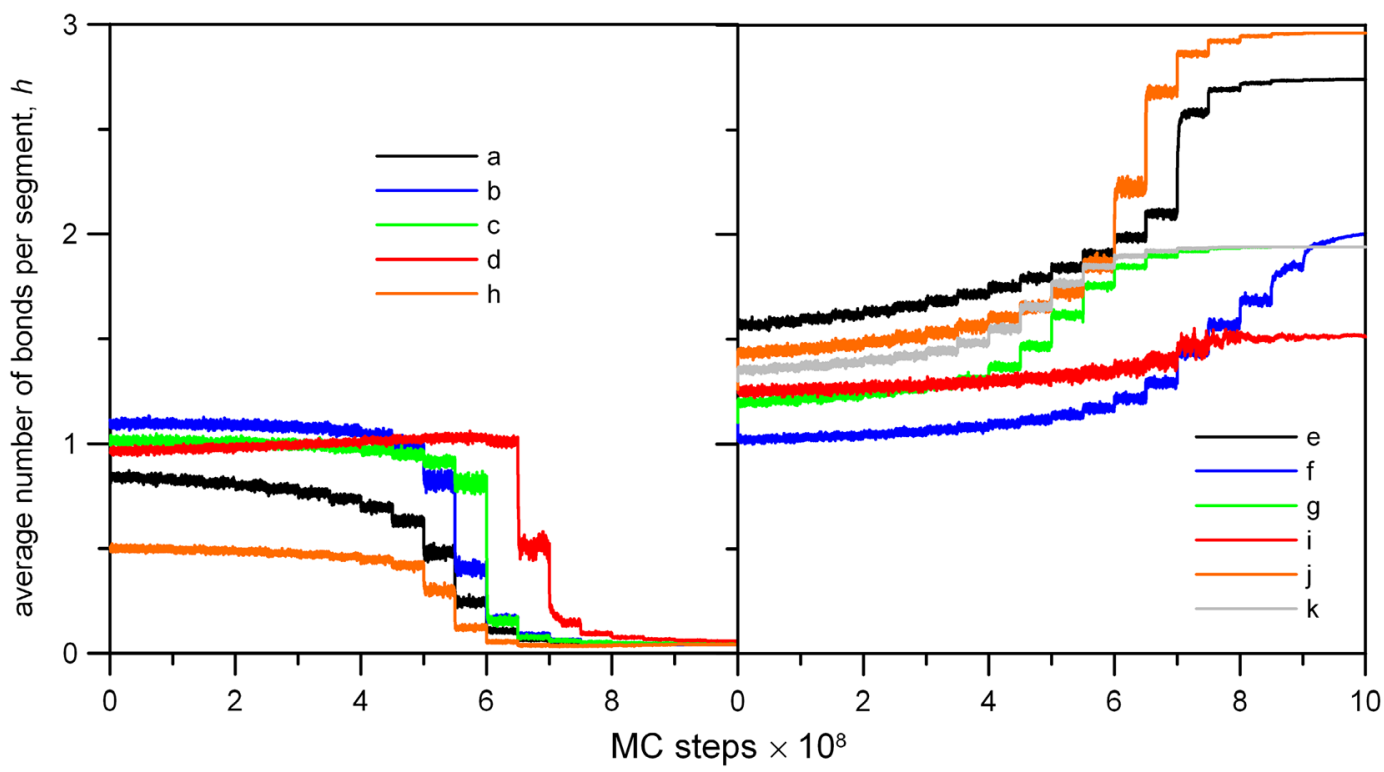

Fig. 7 Changes in the parameter $h$ during the simulation, calculated for the mixtures a-k comprising 650S $+650 \mathrm{R}$ enantiomers

one (2.2). In this case the main reason is the partial demixing which produces homochiral domains and lowers $h$.

To find out the relation between shape of the building blocks from Fig. 1 and their ability to segregate in 2D, for the molecules $\mathbf{a}-\mathbf{k}$ we calculated the corresponding radii of gyration. The radius of gyration is defined as

$R_{\mathrm{g}}^{2}=\frac{1}{N} \sum_{k=1}^{N}\left|\mathbf{r}_{k}-\mathbf{r}_{c}\right|^{2}$

where $N$ is the number of segments in a molecule and $\mathbf{r}_{c}$ is the mean position of the segments (center of mass). This parameter, known from polymer science, characterizes the distribution of molecular segments with respect to the center of mass of the molecule $\left(\mathbf{r}_{c}\right)$. It takes larger values for branched molecules while it is smaller for compact structures. Table 1 collects the obtained values along with the corresponding phase behavior.

The values from Table 1 indicate that, in general, the chiral segregation occurs for those molecules which are characterized by $R_{\mathrm{g}}$ that is larger than about $1.2(\mathbf{a}, \mathbf{c}, \mathbf{h})$. On the other hand, the racemic mixing takes place when $R_{\mathrm{g}}$ is lower than this value, however, with two exceptions including molecule $\mathbf{b}$ and $\mathbf{d}$ for which the resolution was 
Table 1 Correlation between the radius of gyration and the ability of enantiomers $\mathbf{a}-\mathbf{k}$ to segregate in the $2 \mathrm{D}$ racemic overlayers

\begin{tabular}{|c|c|c|c|c|c|c|c|c|c|c|c|}
\hline Type & $\mathbf{a}$ & $\mathbf{b}$ & c & d & e & $\mathbf{f}$ & $\mathbf{g}$ & $\mathbf{h}$ & $\mathbf{i}$ & $\mathbf{j}$ & $\mathbf{k}$ \\
\hline$R_{\mathrm{g}}$ & 1.327 & 1.131 & 1.280 & 0.959 & 1.020 & 1.020 & 1.077 & 1.265 & 1.131 & 1.149 & 1.040 \\
\hline Phase behavior & $s$ & $s$ & $s$ & $s$ & $m$ & $m$ & $m$ & $s$ & $s / m$ & $m$ & $m$ \\
\hline
\end{tabular}

$s$ segregation, $m$ mixing

observed. The obtained results demonstrate that the $R_{\mathrm{g}}$ factor has a limited applicability and can be used as an auxiliary descriptor in the prediction of the chiral resolution. In fact, the ability of the molecules to mix or segregate has a more subtle origin which relates to the shape and in this case other topological descriptors (for example, topological indices) can be more useful. In general the segregation will occur for those molecules which are elongated, resembling rods and have minimal number of kinks. For the molecules which are more compact, ramified and have multiple bends one should expect mixing. However, to asses individual tendency of each molecule the MC simulation approach proposed here seems to be the most informative way.

\section{Conclusions}

In summary, in this contribution we demonstrated that the simple MC lattice model can be effectively used to predict structure of adsorbed overlayers comprising prochiral molecules with diversified shape. The calculations performed for the racemic mixtures of the five-membered probe tectons showed that shape anisotropy is the main factor which enhances the orientationaly confined segregation. The 2D chiral resolution was observed for the molecules with the largest aspect ratio, that is for elongated rod-like shapes with a few (3 or 4) collinear segments. On the other hand, the molecules with more compact shapes or those with ramified structure revealed to be able to form mixed crystals with overall racemic composition. In this case, due to shape factor and intermolecular interactions the surface enantiomers exhibited tendency to form mixed heterochiral pairs instead of homochiral pairs, leading to the ordered mixed phase.

The findings described in this work can be helpful in preliminary selection of molecular building blocks able to segregate and produce enantiopure domains under the orientational confinement. In particular, these hints can substantially reduce the number of test syntheses needed to select the optimal structural unit, for example out of polyphenyl-type molecules. Moreover, it was shown that depending on the molecule at play one can trigger the ordering which can lead either to enantiomer segregation or to mixed crystals. These structural transformations can be potentially used in the fabrication of stimuli-responsive materials able to change their properties under directional magnetic or electric field.

Open Access This article is distributed under the terms of the Creative Commons Attribution 4.0 International License (http://crea tivecommons.org/licenses/by/4.0/), which permits unrestricted use, distribution, and reproduction in any medium, provided you give appropriate credit to the original author(s) and the source, provide a link to the Creative Commons license, and indicate if changes were made.

\section{References}

Ahuja, S.: Chiral Separations by Chromatography. Oxford University Press, Washington, DC (2000)

Berg, A.M., Patrick, D.L.: Preparation of chiral surfaces from achiral molecules by controlled symmetry breaking. Angew. Chem. Int. Ed. 44, 1821-1823 (2005)

Kudernac, T., Lei, S., Elemans, J.A.A.W., De Feyter, S.: Twodimensional supramolecular self-assembly: nanoporous networks on surfaces. Chem. Soc. Rev. 38, 402-421 (2009)

Martínez-González, J.A., Chapela, G.A., Quintana-H, J.: Spontaneous chiral resolution in two-dimensional systems of patchy particles. J. Chem. Phys. 140, 194505-1-194505-5 (2014)

Medved', I., Trník, A., Huckaby, D.A.: Chiral segregation in three microscopic statistical-mechanical models. Phys. Rev. E. 80, 011601-1-011601-7 (2009)

Medved', I., Trník, A., Huckaby, D.A.: Chiral segregation, an unusual racemic phase, and a residual entropy for a lattice system of model chiral molecules. J. Stat. Mech. 12, P12027+14 (2010)

Medved', I., Huckaby, D.A., Trník, A., Milas, I.: Chiral discrimination for a system of tetrahedral molecules on a triangular lattice. J. Stat. Mech. 20151, P06020+12 (2015)

Miyake, K., Hori, Y., Ikeda, T., Asakawa, M., Shimizu, T., Sasaki, S.: Alkyl chain length dependence of the self-organized structure of alkyl-substituted phthalocyanines. Langmuir 24, 4708-4714 (2008)

Mougous, J.D., Brackley, A.J., Foland, K., Baker, R.T., Patrick, D.L.: Formation of uniaxial molecular films by liquid-crystal imprinting in a magnetic field. Phys. Rev. Lett. 84, 2742-2745 (2000)

Mu, Z., Shu, L., Fuchs, H., Mayor, M., Chi, L.: Two dimensional chiral networks emerging from the aryl-F...H hydrogen-bonddriven self-assembly of partially fluorinated rigid molecular structures. J. Am. Chem. Soc. 130, 10840-10841 (2008)

Paci, I., Szleifer, I., Ratner, M.A.: Chiral resolution in two-dimensional racemic systems. J. Am. Chem. Soc. 129, 3545-3555 (2007)

Perusquía, R., Peón, J., Quintana-H, J.: Two-dimensional model for mixtures of enantiomers, bent hard needles: a Monte Carlo simulation. Physica A 345, 130-142 (2005)

Popa, T., Paci, I.: Designing enantioselectivity in chiral self-assembly at a solid substrate: a theoretical study of competing interactions. Soft Matter 9, 7988-7998 (2013) 
Ramirez-Pastor, A.J., Nazarro, M.S., Riccardo, J.L., Zgrablich, G.: Dimer physisorption on heterogeneous substrates. Surf. Sci. 341, 249-261 (1995)

Rampulla, D., Gellman, A.J.: Enantioselectivity on Surfaces. In: Schwarz, J.A., Contescu, C.I., Putyera, K. (eds.) Dekker Encyclopedia of Nanoscience and Nanotechnology, pp. 1113-1123. Marcel Dekker Inc, New York (2004)

Romá, F., Ramirez-Pastor, A.J., Riccardo, J.L.: Multisite occupancy adsorption: comparative study of new different analytical approaches. Langmuir 19, 6770-6777 (2003)

Szabelski, P., Woszczyk, A.: Role of molecular orientational anisotropy in the chiral resolution of enantiomers in adsorbed overlayers. Langmuir 28, 11095-11105 (2012)

Tahara, K., Yamaga, H., Ghijsens, E., Inukai, K., Adisoejoso, J., Blunt, M.O., De Feyter, S., Tobe, Y.: Control and induction of surface-confined homochiral porous molecular networks. Nat. Chem. 3, 714-719 (2011)

Woszczyk, A., Szabelski, P.: Enhancing the separation of enantiomers in adsorbed overlayers: a Monte Carlo study. Ann. UMCS 68, 133-141 (2013)

Woszczyk, A., Szabelski, P.: Theoretical investigations of the 2D chiral segregation induced by external directional fields. RSC Adv. 5, 81933-81942 (2015)

Xu, H., Ghijsens, E., George, S.J., Wolffs, M., Tomović, Z., Schenning, A.P.H.J., De Feyter, S.: Chiral induction and amplification in supramolecular systems at the liquid-solid interface. ChemPhysChem 14, 1583-1590 (2013)

Zaera, F.: Chiral modification of solid surfaces: a molecular view. J. Phys. Chem. C 112, 16196-16203 (2008) 